\title{
PROSPECTS FOR MALARIA CONTROL
}

\author{
Pierre Carnevale ánd Jean Mouchet
}

Institut Français de Recherche pour le Développement en Cooperation, ORSTOM, 213, Rue La Fayette 75010 Paris. France.

\section{INTRODUCTION}

Malaria was considered as one of the main health problems in the world and its control was actively undertaken after the Second World War. Impressive success has been obtained in a number of countries, mainly in the most developed une:s. Nevertheless malaria is still common in the tropical world and several hundreds of millions of people are at risk. In 1977 more than 10 million cases were reported to the World Health Organization (WHO) (World Health Statistics, quart; 38, 1984). This number is largely underestimated; most cases go unreported. and Africa, the biggest malarious area in the world, was not included in the statistics. World Health Assemblies in recent years have stressed the urgency of reducing the prevalence of malaria, not only as a health problem but also as a serious impediment to socio-econumic development. Even if existing control technics are not perfect they would allow serious progress in malaria control if applied properly everywhere. In this paper we highlight the problems associated with the application of existing technics rather than the purely technical questions which have already received a lot of attention.

\section{OBJECTIVES AND STRATEGIES OF MALARIA CONTROL}

It was recognized in 1968 that eradication could not be attained everywhere. Control then became the aim of malaria activities in countries not involved in eradication programmes. But a clear definition of objectives, strategies and tactics of such control took some time to emerge as most malariologists were still upset by the eradication dogma. The Interregional Conference held in Brazzaville in 1972 (WHO, 1974a) only recommended more research, the training of qualified personnel and an approach of community participation. The I6th Expert Committee on Malaria (WHO. 1974b) defined the objectives of malaria control as a reduction of mortality and morbidity with a subsequent improvement in socioeconomic development. Following the conclusions of a group of WHO Experts (WHO. 1984). the 38th World Health Assembly recommended that antimalaria activities should be integrated into the Primary Health Care (PHC). System (Decision WHA. 38.24) with community participation to cover all the population at risk and avoidance of concentration of sophisticated care on small groups.

The great variability of malaria epidemiology as well as the marked differences in resources available for controlling the disease from one country to another (WHO, 1979) preclude any attempt to draw up a global strategy of malaria control (Najera, 1985, unpublished document). Strategies should be established locally according to epidemiological determinants, socio-economic features and available resources. Therefore it is urgent to develop sound epidemiological studies including socio-economic factors to establish in each country a malaria stratification. The planning of antimalaria measures has to be established for exich stratum. Activities have to be evaluated on a permanent basis not only to detect failures but also for acquiring experience to improve the overall system. Then it will become easier to follow the evolution of the malaria situation according not only to control measures but also to socio-economic and environmental modifications. Carrying out such activities requires the availability at the central level of a team of highly qualified personnel including epidemiologists, entomologists, sanitary engineers, specialists in social science, economy and demography with special training in malaria. They will have to collect the basic information, to plan the activities and later to provide guidance to health authorities and to proceed with evaluation of activities.

Well trained officers are also necessary at the intermediate level to make the link between the central level and the PHC agents and for providing them with direct guidance and supervision. 


\section{TACTICS}

In 1978 four tactical variants were presented at the 31 st World Health Assembly and later endorsed by the 17th Expert Committee on Malaria (WHO, 1979).

1) Reduction/prevention of mortality due to malaria by case treatment.

2) Reduction/prevention of mortality and morbidity by drug administration and protection of highly vulnerable groups.

3) Administration of drugs and vector control measures to prevent mortality and morbility and to reduce prevalence.

4) Application of control measures on a countrywide basis to possibly eliminate the disease.

As pointed by Beales \& Najera (1985, unpublished document) there is no clear cut distinction between these variants. In fact there is a continuum from the simple treatment of an acute case to the interruption of transmission in the whole country. Moreover other technics can be incorporated in the variants like personnel protection in the variant $\mid$ and 2.

The first two variants are dealing with case treatment and chemoprotection, the last two with decrease or interruption of transmission through vector control, leading to eradication. In fact the word eradication is wrong as it means "remove the roots". Now unless there is a drastic change in the malaria equilibrium or in vector bionomics, the "roots" remain during the malaria eradication process and the malaria epidemiological complex is able to rebuild when the control ceases as the vectors are still there and the parasite can at any time be reintroduced from a malaria-free area. Such events have been observed in several countries in South East Asia. Therefore before undertaking variants 3 and 4 , countries must be aware that it is a long term process unless a change of malaria equilibrium is expected.

\section{Treatment of malaria cases}

\section{TECHNICS AND METHODS}

Case diagnosis and treatment are the basis of malaria control. Therefore diagnostic facilities should be expanded through the PHC system. If these facilities are not yet available, as in most African villages, presumptive treatment of febrile cases suspected to be malaria could be undertaken mainly if relatively safe drugs like chloroquine can be used (Jeffery, 1984).

Drug resistance of Plasmodium falciparm is expanding rapidly and is a serious threat for chemotherapy and chemoprophylaxis. Chloroquine resistance, widespread in Asia, is well established in America and East Africa and has been recently recorded in central Africa. But in most of Africa the parasite remains susceptible or shows only a decrease in susceptibility. Resistance to another amino-4-quinoline, amodiaquin, follows the same pattern. It has been known since 1954 that resistance to pyrimethamine was developing quickly in areas where this drug was used mainly for chemoprophylaxis; it is probably responsible for resistance to the combination of pyrimethamine with sulfadoxine quite frequently encountered in South East Asia and reported from Easi Africa. Resistance to quinine seems related to chloroquine resistance (Onori, 1984).

The choice of a drug for treatment and its posology depends on the parasite susceptibility. Therefore surveillance of drug susceptibility should be an integral part of antimalaria activities.

In areas where the susceptibility to chloroquine is reduced suppressive treatment should be increased from a single dose of chlorequine of $10 \mathrm{mg} / \mathrm{kg}$ of body weight to a dosige of $25 \mathrm{mg} / \mathrm{kg}$ of body weight in 3 days. If the strain is resistant other medicament. like quinine or combination of pyrimethamine and sulfadoxine (Fansidar $R$ ), have to be used if the strains respond to them.

A new compound, mefioquine, is now available. It can be taken alone or in triple combination with pyrimethamine and sulfadoxine (Fansimef $R$ ) which permits suppressive treatment of a resistant strain with one dose (Fernex, 1985). WHO has strongly recommended to reserve mefloquine and Fansimef for therapy and not to use it in chemoprophylaxis to avoid the selection of resistance which has already been obtained in animal models.

Qinghaosu, an active compound from Artenmisia armua used in traditional Chinese medicine. is in the process of development. It is fast acting and very rapidly excreted. It is mainly used in China for treatment of cerebral malaria. Piperaquine used in China does not show advantages over chloroquine (Dobertsyn, 1985, unpublished WHO document). Halofantrin is in the advanced stages of development.

Primaquine remains the only sporonticide available. It is used for suppression treatment of vivar malaria. It has been proposed that it should be used for treatment of resistant falciparmm, to kill gamecocytes and avoid the spread of resistant parasites. But more information is needed on its toxicity, mainly for those who are deficient in G6PU.

PHC systems are probably the best channels for drug delivery and case treatment. And these activities can in turn be the starting point for PHC development. In several countries drug delivery and case 
treatment have already been organized for many years through antimalaria structures. Whenever these structures have to be integrated to PHC depends on the responsibility of local health authorities. The important thing is that efficient medication reaches the peripheral level and is accurately delivered.

Early treatment of malaria cases reduces the probability of more severe symptoms. In remote hamlets or in places where PHC is not yet fully efficient it can be difficult to obtain drugs rapidly for treatment. Then it might be necessary to leave a provision of medicines to a community representative or better to the mothers. Of course all those who have to use drugs must be fully taught of the treatment procedure mainly if compounds other than chloroquine have to be used. In any case patients have to be shown to health officers or PHC agents as soon as possible.

Our experience in Central and West Africa has shown that in the last 20 years mortality due to malaria has been reduced by the self-use of chloroquine by the population, the drug being channeled through the health system or traders. In many areas the mothers used to give chloroquine to their children when "iheir body is warm ". It is difficult to estimate the impact of such practices but we were impressed by the small number of cerebral cases and of death attributable to malaria in holoendemic areas where no. specific antimalaria measures are undertaken as in the Congo. (Collective paper, 1982).

\section{Preventive measures}

There is a consensus for the chemoprophylaxis of pregnant women (WHO, 1979) but more debated is the prophylaxis of infants and children below five because of the risk of side-effects of continuous use of drugs for several years (Salako, 1984) and of selection of resistant strains of parasites (Jeffery, 1984). Also a countrywide programme of prophylaxis would be costly, and would require a strong organization above the capabilities of most of the PHC systems. Moreoever attendance of healthy children at regular drug distributions decreases rapidly and a great proportion of them will remain without any protection (Laing, 1984). It has been observed in Burkina Faso and in Nigeria a decrease of antibody rate in children under prophylaxis (Baudon, 1984, pers. comm.; Greenwood, 1984); these observations call for deeper investigations.

Chemoprophylaxis remains the best way to protect non-immune people who stay for a limited time in endemic areas. The choice and the regimen of prophylactic drugs depends, of course, of the susceptibility of local strains of parasites.

\section{Vector Control}

Residual house-spraying. Reduction or break-down of the parasite's transmission by house-spraying with residual insecticides was the basis of malaria eradication. It remains in many countries an important if not the main component of malaria control. Where DDT resistance made the treatments ineffective, turning to organophosphates (O.P.) or carbamates increased the cost of operations several fold and some countries could not afford such extra expenses. Multiresistance to DDT and to OP and/or carbamates has already been reported from several countries (WHO, 1980). Generally malathion resistance is not linked with other O.P. or carbamates and does not preclude the use of fenitrothion and propoxur. Up to now it remains possible to control anopheline vectors by one compound or another even if it is difficult in some areas such as the Pacific Coast of Central America. But in some places e.g. in Turkey, the population was reluctant to accept house spraying.

The value of house-spraying, now in use for more than thirty years, has been analysed in several countries. If it has not lead to eradication it has drastically reduced malaria. However, in a number of areas its objective, intertuption of transmission, could not be reached. Lack of coverage and resistance to insecticide are the two main reasons advanced to explain these failures. But the behaviour of both men and vectors is also responsible for a number of problems all over the world. Now more attention is paid to population movements, migration and behaviour as well as to the exophily of anophelines, eventually enhanced by the irritating effect of some insecticides.

Only five per cent of insecticide production is employed for vector control. Consequently not a single compound-either larvicide (except Bt $\mathrm{H}(4)$ ) or adulticide - has been developed for the special purpose of public health. WHO has built a pesticide development scheme where nearly all the insecticides produced by the chemical industry (more than 3000 ), have been tested for their potential in vector control. The cost of develepment of compounds has increased considerably in the last few years mainly due to security constraints. Consequently the number of new products available for testing has sharply decreased.

For house spraying, only two O.P., pirimiphos methyl and chlorphoxim, and one carbamate, Bendiocarb, have been recentiy tried operationally and their performances were in the same range as fenitrothion and propoxur, already in use (Panl, Rishikesh \& Smith, 1985, unpublished document).

Synthetic pyrethroids-permethrin. deltamethrin, cypermethrin and alphametrin-show very high insecticidal activity but have an adverse repellent effect. It would be desirable to evaluate these 
compounds at a village scale to observe if their repellent effect does not cuntribute to the disruption of man-vector contact and the overall impact of the treatment on the disease.

House spraying is generally carried out by specialized teams belonging to vertical structures. Here too it is governments to decide if they want to transfer these activities to PHC systems and how they want to do it. Already in several countries of Asia. house spraying is totally or parlly done by volunteer or paid members of communities under the guidance and supervision of more specialized personnel. So far the results seem to be satisfactory.

To reduce the consumption of insecticide and manpower, limited selective treatments have been carried out in Indonesia. Spraying only the lower part of the walls below $1.5 \mathrm{~m}$ with fenitrothion reduced the malaria rate and vector population (Anopheles uconitus) to the same extent as total coverage but less rapidly (Gandahusada et al., 1984). Good control of the same vector species could be obtained by treating only animal shelters with fenitrothion or with pirimiphos methyl (Shaw, Barodji, Fleming, Pradhan \& Bang, 1983; Barodji, Shaw, Pradhan, Bang \& Fleming, 1984).

Integrated vector control. The principle of integrated control is to use together all the available technics and tools to control the vectors. It is understood that the integration of environmental and biological methods will reduce the consumption of insecticides. The available methods and technics have been reviewed by a WHO Expert Committee (WHO, 1983) which has moreover emphasised community involvement in this kind of control.

Integrated control has been a magic word, able to solve all vector control problems. In fact it is not a simple task and not at all a panacea. Most of the methods recommended for integration like source reduction, larval control by fishes, and water management have been used during the pre DDT era with limited success. The feasibility and efficiency of control measures depends of vector ecology. For example, in a semi-desert area of Somalia where An. arabiensis was breeding only in underground tanks, the fish Oreochromis spilurus gave almost complete control of both vectors and malaria (Alio \& Isaq, 1982). But in other places in Africa where the same mosquito species and the sibling An. gambiae develop in temporary rainpools, fishes would be of little help for control. The same could be said about source reduction and water management, sometimes easy, sometimes impossible. Moreover, control is made on larval stage which implies that all breeding places should be treated or suppressed because the mosquitoes emerging have free access 10 malaria transmission. To treat or to suppress the breeding sites it is necessary to find them, which is not always easy (e.g. for An. dirus in the jungle). Treatments with chemicals or with bacterial preparations needs to be rrequently repeated as their residual effect is short. For example Bacillus thuringiensis $\mathrm{H} / 4$ is very toxic for anophelines and very safe for man and the environment but the residual effect of the availabie formulations is only 24 hours, and a weekly application would be necessary to keep the pools free of pupae (Hougard, Darriet \& Bakayoko. 1983). These examples have been chosen to draw attention to the complexity of integrated vector control. More than any antimalarial activity it has to be locally adapted. Appropriate technics and methods should be selected for each area, sometimes for . each village according to ecological factors and population behaviour. Such an approach needs al least the collaboration of a highly qualified entomologist with an epidemiologist and a specialist in social sciences. There must be a sound evaluation of all activities to assess their impact on the vectors and on the disease as well as their acceptance by the local population.

The other magic word is community participation which is rather imprecise. In the case of malaria vector control, which is a long term and varied activity, there is, at least. two levels of involvement of the community: conmunity agents, permanent or temporary, with an appropriate training ate required for spraying. larviciding, fish stocking and all time-consuming work. As it is a long term job they have to be paid by somebody (either health services or community) and recruitment of volunteers is rather improbable. Activities of environmental sanitation have to be done by every member of the community. They have to be careful not to discourage people. Appropriate health education is the best way lo motivate the community.

But community participation will be maintained only if community members perceive the beneficial effects of their efforts. In this respect suppression of the nuisance activity of biting insects is a great incentive and should be taken into consideration in the planning (Mouchet. 1982).

In Gujerat State, India, an experimental integrated vector control project with community participation has been running for two years in 8 villages. It was successiul in reducing the vector density and subsequently the number of malaria cases by source reduction, environmental managenent and larvivorous fishes. It must be underlined that it had good scientific support (Rishikeesh. 1985. Pers. comm.). Nevertheless it is dillicull to anticipate what will become of such a project if it is integrated into general nublic health activities.

Individual protection. Mosquito nels are currently used for self protection. It is the most simple way to break man-vector contact. But very often bed nets are wrongly placed especially where people sleep on 
mats. Moreover the maintenance of the nets is often poor and when they are perforated they do not work properly. Impregnation of bednets with knock-down insecticides e.g. pyrethrinoids was thought to overcome these difficulties. Trials made with permethin in Burkina Faso (Darriet, Robert, Tho Vien \& Carnevale, 1984) and in Tanzania (Lines, Curtis, Myamba \& Njau, 1985) were very promising. Large scale experiments with nets impregnated with permethin in Papua New Guinea and with deltamethrine in China are under epidemiological evaluation (Schrek \& Self, 1985, personal comm.; Ho \& Li, 1985, pers. conım.). To a certain extent impregnated nets can be considered as equivalent of selective spraying and have to be evaluated as such.

Private use of mosquito coils and fumigation mats are very popular to avoid the mosquito nuisance. They reduce the risk of indoor Iransmission (Smith \& Obudho, 1967).

\section{Vaccination}

Great hopes are held for a malaria vaccine but it is not yet possible to anticipate its place in malaria control strategies. It will depend on the type of vaccine, the duration of the protection, its relationship to natural immunity, its conditions of conservation and of epidemiological conditions etc. But field trials of any vaccine development imply a sound knowledge of local epidemiological determinants in the test area. Such studies could become methodological models for other epidemiological investigations.

\section{INTERSECTORIAL ACTIVITIES}

Development schemes strongly modify the environment. It is well known that irrigation is a source of mosquito breeding and very of ten but not always an increase in malaria. Heaith authorities and developers have to plan together any measure which would be able to minimize the adverse impact of the new infrastructures, both during their building and later during their functioning. Non-immune immigrants have also to be protected. But on the other hand development increases income and people become able to afford their own or collective protection.

As vector resistance is very often selected by agricultural pesticides, Sri Lankan authorities have taken the interesting initiative of banning malathion from agriculture, reserving its use for public health.

\section{TKAINES}

As malaria control should be organized on a lacal basis there is an increased need for expertise to stratify malarious areas, plan operations and evaluate their impact and adequacy. A sound knowledge of malaria should be given not only to the epidemiologisis but also to the entomologists, sanitary engineers and specialists in social sciences. A multidisciplinary approach implies that all the scientists involved have the same target, can understand each other and work on the same canvas. The necessity of field training has to be underlined.

The training of PHC agents has to be concentrated on the tasks they will have to perform. They can be specialized for malaria control or have a multipurpose activity depending of the PHC local organization.

Health education should be adapted to the local situation and a particular effort should be made to reach the mothers who can be considered as the primary PHC agents.

\section{RESEARCH}

Rmong all the numerous research subjects proposed in every meeting we would just call attention to those which have immediate relevance for implementation of malaria control with existing technics and tools. The following list is not at all exhaustive. The needs for new medication and new insecticides and for better use of those already available has been continuously stressed.

Epidemiological research is essential for the stratincation of malaria, the planning, the implementation and the evaluation of control measures defined on a local basis. It implies:

- The evaluation of the load of the disease (and not only the parasitic infection) with adequate criteria

- the study of transmission patterns and the ecology and biunomics of vectors

- the immune status of the population

- the socio-economic determinants

- the willingness of the community to participate and the incentive measures to enhance this participation

- the available resources for control.

A simple diagnostic tool is urgently needed for peripheral units where microscopic facilities are not available. 
There is a great need for research to produce appropriate technics for integrated vector control and for individual protection and to adapt those already existing to different ecological situations. The impact of integrated control measures and of individual protection (mainly impregnated nets) has to be evaluated on the disease itself as well as on its vectors.

Surveillance of susceptibility of parasites to drugs, and of vectors to insecticides, is becoming a routine activity but development of more simple methods is a research target.

It is to be expected that the rapid progress in biotechnology and genetic engineering will provide new tools for malaria control in the near future.

\section{SUMMARY}

Malaria control strategies have to be established locally according to epidemiological situations, including socio-economic factors and to resources available for their implementation. It has been stressed that all antimalaria activities be integrated in PHC.

Stratification of malaria is the introduction to malaria conirol and serves as a basis for the planning which should be established by a body of experts on malariz at the central level (epidemiologist, entomologist, specialists in social sciences, sanitary engineer) who later will guide, supervise and evaluate the activities.

Case treatments, sometimes presumptive, are the most basic activities of control. They are cheap and they can be carried out by PHC which insures the coverage of the entire population at risk. Drug resistance of Plasmodium falciparum is a growing and threatening problem. In a number of areas cheap and harmless chloroquine has to be replaced by drugs or combinations of drugs which can only be delivered by experienced personnel. Chemoprophylaxis is recommended for pregnant women but questioned for infants and young children because the risk of side-effects and resistance selection and the difficulties of maintaining a good coverage for a long time.

Vector control by house-spraying remains the best means of reducing transmission and is still the basis of malaria control in countries in Asia and America where the disease has been seriously reduced. In some areas resistance to DDT lead to the use of more expensive organophosphates andior carbamates. Resistance to these compounds has also been reported in several countries. House-spraying is probably one of the malaria activities which is the most difficult to integrate in PHC. Some attempts have been successful.

Integrated vector control with community participation is not a simple task nor a panacea. To be efficient it needs to be established on a strong scientific basis. Tools and technics have to be selected for each area according to the vector ecology and socio-cultural habits of the population. Maintaining community interest in a long lasting activity is a problem which has never been really explored. Self-protection against vectors (mainly by using impregnated mosquito nets) has shown promise and is currently being evaluated at an operational scale with community involvement. There is great hope for a vaccine but more advances are necessary before its place in malaria control can be established.

Intersectorial approach is the best way to counteract undesirable effects of development schemes like irrigation. There is a need for training in the scope of interdisciplinary actions for high level personnel. PHC agents need special training whatever they are, specialized or multipurpose. Special attention must be paid to malaria in health education for communities at risk. Some researches dealing with the most immediate problems are suggested.

\section{REFERENCES}

AL1O, A.Y. \& ISAQ, M.A. 1982. Field trial on the impact of Oreochromis spilurns on malaria transmission in Northern Sonnalia. WHO.mimcog. doc. WHO/EM/Mal./I9I.

Barodi, Shaw R.F., Pradhan, G.D., Bang Y.H. \& Fleming G.A. 1984. Communily participation in the residual treatment of cattle shelters with pirimiphos methyl (OMS 1424) to control a zoophilic malaria vector Anophele aconitus; a village scale trial. WHO. mimeog. doc. WHO/VBC/84.897.

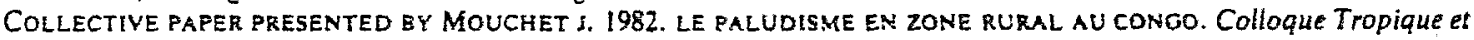
Santé CEGET Bordeaux ed.. 109-119.

DARRIET F.. ROBERT V., ThO VIEN \& CAENEVALE P. (1984). Evaluation of the efficacy of permethrin-impregnated intaçtand perforated mosquito nets against vectors of malaria. WHO. mimeog. doc. WHO/VBC/84.899.

FERNEx M. 1985. Mefloquine and its allies. World Health. May 1985: $6-7$.

Gandahusada S., Fleming G.A.. Sukmato. Dakiar T.. Suwhrto. Suskriaya K., Bang Y.H.. Arwati F.S. \& ARIF H. 1984. Malaria control with residual fenitrothion in Central Jeva, Indonesiz: an operational scale trial using both full and selective coverage ireatments. Bulletin of the Wortd Heath Organization 62: 783-794.

GREENWOOD B.M. 1984 . The impact of malaria chemoprophylaxis on the immune status of Africans. Bulletin of the World Health Organization 62 (suppl.): 69-75. 
Hougard J-M.. Darriet Fr. \& Bakayoko S. 1983. Evaluation en milieu naturel de l'activité larvicide de Bacillus thuringiensis serotype H 14 sur Culex quinquefasciatus et Anopheles gambiae en Afrique de l'Ouest. Cahiers ORSTOM, série Entomologie médicale et Parasitologie 21: $111-117$.

JEFFERY G.M. 1984. The role of chemotherapy in malaria control through primary health care: constraints and future prospects. Bulletin of the World Health Organization 62 (suppl.): 49-55.

LAiNg A.B.C. 1984. The impact of malaria chemoprophylaxis in Africa with special reference to Madagascar. Cameroon and Senegal. Bulletin of the World Health Organization 62 (suppl.): $41-49$.

LINES J.D., CURTIS C.F., MYAMBA J. \& NJAU R. 1985. Tesis of repellents or insecticide impregnated curtains, bednets and anklets against malaria vectors in Tanzania. WHO mimeog. doc. WHO/BVC/85.920.

MOUCHET J. 1982. Vector control at community level. WHO. mimeog, doc. WHONVBC/82.847.

ONORI E. 1984. The problem of Plasmodium falciparum drug resistance in Africa south of the Sahara. Bulletin of the World Healtil Organization 62 (suppl.): $55-63$.

Salako L.A. 19H4. Tuxicity and side-effects of antimarials in Africa; a critical review. Bulletin of the World Heally Organization 62 (suppl.): $63-67$.

Shaw R.F.. Barodit, Fleming G.A.., Pradhan G.D. \& Bang Y.H. 1983. Residual treatment of cattle shelters using fenitrothion to control a zoophilic malaria vector Anopheles uconitus in Indonesia: A village scale trial. WHO mimeog. doc. WHONBC/83.877.

Sмiтн A. \& Овионо W.O. 1967 . Trials with pyrethrum mosquito coils against Anopheles gambiae. Mansonia uniformis and Culex fatigans entering verandah trap huts. Pyrethrum Post 11 i $111-113$.

WHO 1974a. Lutte contre le paludisme dans les pays où un programme d'éradication limité dans le temps n'est past realisable actuellement. WHO Technical Report Series n' 537 .

WHO 1974b. Expert Committee on malariz. Sixteenth Report. WHO Technical Report Series n'549.

WHO1979. Expert Commiltee on malaria, Seventeenth Report. WHO Technical Report Series n'640.

WHO 1980. Resistance of vectors to pesticides. WHO Techmical Reporn Series n'655.

WHO 1984. Malaria Control as part of Primary Health Care. WHO Technical Report Series n'7l?. 


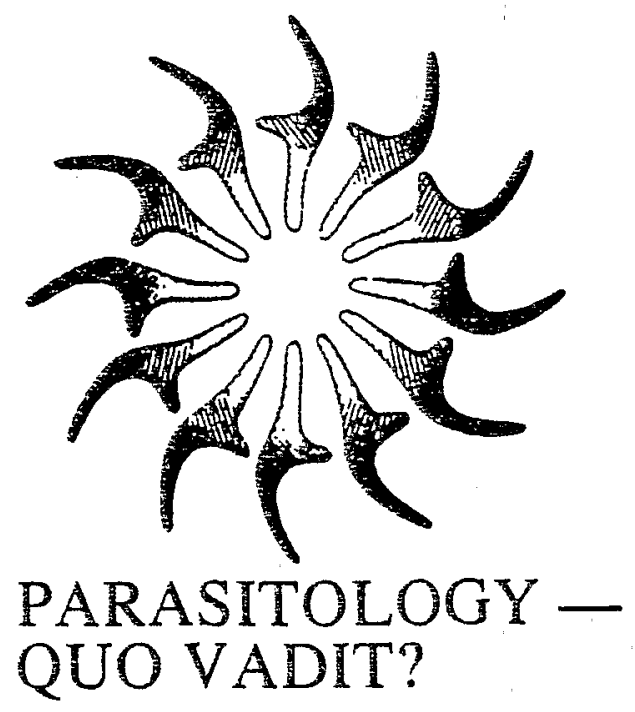

PROCEEDINGS OF THE

SIXTH INTERNATIONAL

CONGRESS OF

PARASITOLOGY

Edited by

M.J. Howell,

Department of Zoology,

Australian National University,

Canberra, A.C.T.,

Australia. 